\title{
SHARP ASYMPTOTICS IN A FRACTIONAL STURM-LIOUVILLE PROBLEM
}

\author{
Pavel Chigansky $^{1}$, Marina Kleptsyna ${ }^{2}$
}

\begin{abstract}
The current research of fractional Sturm-Liouville boundary value problems focuses on the qualitative theory and numerical methods, and much progress has been recently achieved in both directions. The objective of this paper is to explore a different route, namely, construction of explicit asymptotic approximations for the solutions. As a study case, we consider a problem with left and right Riemann-Liouville derivatives, for which our analysis yields asymptotically sharp estimates for the sequence of eigenvalues and eigenfunctions.
\end{abstract}

MSC 2010: Primary 34B24, 26A33; Secondary 60G22

Key Words and Phrases: fractional calculus; Sturm-Liouville problems; asymptotic approximation

\section{Introduction}

The theory of boundary value problems of Sturm-Liouville type is a current subject of research in fractional calculus and its applications. A number of formulations have been considered, corresponding to various nonequivalent types of fractional derivatives and motivated by different applications. Beyond a few special cases, such as e.g. [30], these problems do not have explicit solutions, which leaves much space for qualitative theory, numerical methods and asymptotic analysis.

The research in qualitative theory is concerned with the properties of solutions in problems with different fractional operators and boundary conditions, [15], [18, [9], 21], 19. They can be studied by a variety of tools, including functional analysis methods [22], perturbation techniques [28], variational characterizations [17], integral transforms [8]. Numerical analysis of fractional Sturm-Liouville problems can also be approached in a

(C) Year Diogenes Co., Sofia

pp. $\mathrm{xxx}-\mathrm{xxx}$, DOI: 
number of ways, [2], [3, 44, [20], [25]. These references are only a small part of works on the subject in the recent years.

In this paper we address the problem of asymptotic approximation of solutions to fractional eigenvalue problems. This is a classical theme in functional analysis and mathematical physics, which, in the fractional setting, has not yet been explored in depth. Such approximations have numerous applications, as they determine properties of various physical quantities, see e.g. [11, 23, 5]. Moreover, they can be a decent alternative to numerical methods, which typically remain efficient only for a few dozens of the first eigenvalues and eigenfunctions. Our computational experiments indicate that even in this range asymptotic approximations may provide a very reasonable accuracy, see Figures 1-2 below.

Our main objective is to draw attention to a technique, which can be useful for deriving sharp asymptotic estimates of the eigenvalues and eigenfunctions in fractional Sturm-Liouville problems on a finite interval. As in the analysis of the fractional ODEs on the whole semi-axis, see e.g. [13, it is based on the Laplace transform, however in a completely different way. The crucial property which makes the Laplace transform work on unbounded domains is that it converts the action of fractional operators into multiplication by power functions. This feature is no longer available, when the domain of functions in question is bounded, and instead, the method of this paper makes use of its analytic structure instead.

Our approach is capable of producing uniform approximations for various spectral problems, but the detailed analysis and the ultimate results are quite sensitive to peculiarities of the concrete problem at hand. As our study case, we will consider a basic, yet nontrivial fractional boundary value problem

$$
\begin{aligned}
& D_{1-}^{\alpha} D_{0+}^{\alpha} f(x)=\lambda f(x), \quad x \in[0,1], \\
& f(0)=f(1)=0,
\end{aligned}
$$

with the left and right Riemann-Liouville derivatives of order $\alpha \in\left(\frac{1}{2}, 1\right)$,

$$
\begin{aligned}
& D_{0+}^{\alpha} f(x)=\frac{1}{\Gamma(1-\alpha)} \frac{d}{d x} \int_{0}^{x}(x-t)^{-\alpha} f(t) d t, \\
& D_{1-}^{\alpha} f(x)=\frac{1}{\Gamma(1-\alpha)} \frac{d}{d x} \int_{x}^{1}(t-x)^{-\alpha} f(t) d t .
\end{aligned}
$$

These and other standard definitions and formulas from fractional calculus can be found in, e.g., 13 .

Problem $(\mathbb{P})$ was studied in $[12]$ within the functional analytic framework. The authors prove that the inverse operator is self-adjoint and compact in suitable spaces. Consequently its spectrum is discrete and thus the problem has countably many solutions $\left(\lambda_{n}, f_{n}\right)_{n \in \mathbb{N}}$. The eigenvalues $\lambda_{n}$ are 
real and nonnegative and, accumulate at infinity, and the corresponding eigenfunctions $f_{n}$ are continuous and form an orthogonal basis in the relevant Hilbert space. Alternatively these results can be obtained by means of reduction to the spectral problem for a compact self adjoint integral operator with a certain symmetric continuous kernel, see [16], [19].

\section{Main result}

Our main result is the following theorem, which details asymptotic structure of solutions to the eigenproblem formulated in the previous section.

Theorem 2.1. Let $\left(\lambda_{n}, f_{n}\right)_{n \in \mathbb{N}}$ be the solutions to $(\mathrm{P})$ with $\alpha \in\left(\frac{1}{2}, 1\right)$, ordered so that the eigenvalues $\lambda_{n}$ form a nondecreasing sequence.

(a) The sequence of frequencies $\rho_{n}:=\lambda_{n}^{1 /(2 \alpha)}$ has the asymptotics

$$
\rho_{n}=\pi n+\frac{\pi}{2}\left(1-\frac{1}{\alpha}\right)+O\left(n^{-1}\right), \quad n \rightarrow \infty .
$$

(b) The corresponding eigenfunctions with the unit $L_{2}$-norm satisfy

$f_{n}(x)=\sqrt{2} \sin \left(\rho_{n} x+\frac{\pi}{4}(1-\alpha)\right)$

$+\int_{0}^{\infty} \Upsilon_{0}(t) e^{-\rho_{n} t x} d t+(-1)^{n} \int_{0}^{\infty} \Upsilon_{1}(t) e^{-\rho_{n} t(1-x)} d t+r_{n}(x) n^{-1}, d x \in[0,1]$,

where $\sup _{n}\left\|r_{n}\right\|_{\infty}<\infty$ and $\Upsilon_{0}(t)$ and $\Upsilon_{1}(t)$ are explicit functions (see (3.38) below).

Several comments are in order.

a) Asymptotic approximation of the eigenvalues, implied by this result, is exact up to the second term with a sharp estimate for the residual,

$$
\lambda_{n}=(\pi n)^{2 \alpha}+\pi(\alpha-1)(\pi n)^{2 \alpha-1}+O\left(n^{2 \alpha-2}\right), \quad n \rightarrow \infty .
$$

The first term of this asymptotics can also be derived through reduction to the eigenproblem for an integral operator [16] and the general results in spectral theory [1]. Our approach does not appeal to such a reduction and is based on direct analysis of the fractional operators.

Formula (2.2) implies that, for large $n$, the eigenfunctions behave as pure harmonics, away from the boundary points $\{0,1\}$. The two integral terms form the boundary layer, as their contribution is negligible in the interior of the interval and they force the eigenfunctions to vanish at its endpoints. It should be emphasised that the boundary layer terms are asymptotically negligible with respect to approximation in the $L_{2}([0,1])$ 
norm, but not in the uniform norm. The second order term in the frequencies $\rho_{n}$ is non-negligible for the purpose of approximating the eigenfunctions in $L_{2}([0,1])$ norm.

For $\alpha=1$ problem $(\mathrm{P})$ reduces to the classical problem

$$
\begin{gathered}
-\frac{d^{2}}{d x^{2}} f(x)=\lambda f(x), \\
f(0)=f(1)=0,
\end{gathered}
$$

for which elementary calculations give

$$
\lambda_{n}=(\pi n)^{2} \quad \text { and } \quad f_{n}(x)=\sqrt{2} \sin (\pi n x), \quad n=1,2, \ldots
$$

It can be seen that $\Upsilon_{0}(\cdot)$ and $\Upsilon_{1}(\cdot)$ in (2.2) vanish as $\alpha \rightarrow 1$ and hence these formulas coincide formally with (2.3) .

b) Numerical approximations to solutions of fractional Sturm-Liouville problems, even as basic as problem $(\mathbb{P})$, typically provide good accuracy for a few first eigenvalues and eigenfunctions, but become unstable already for $n \geq 30$. The approximation provided by Theorem 2.1 can thus be a reasonable alternative. Our numerical experiments indicate that formulas (2.1)-(2.2) with residuals being truncated, turn out to be quite accurate, at least beyond several first eigenpairs, see Figures 1-2. They also demonstrate significant improvement due to the second order term in (2.1).

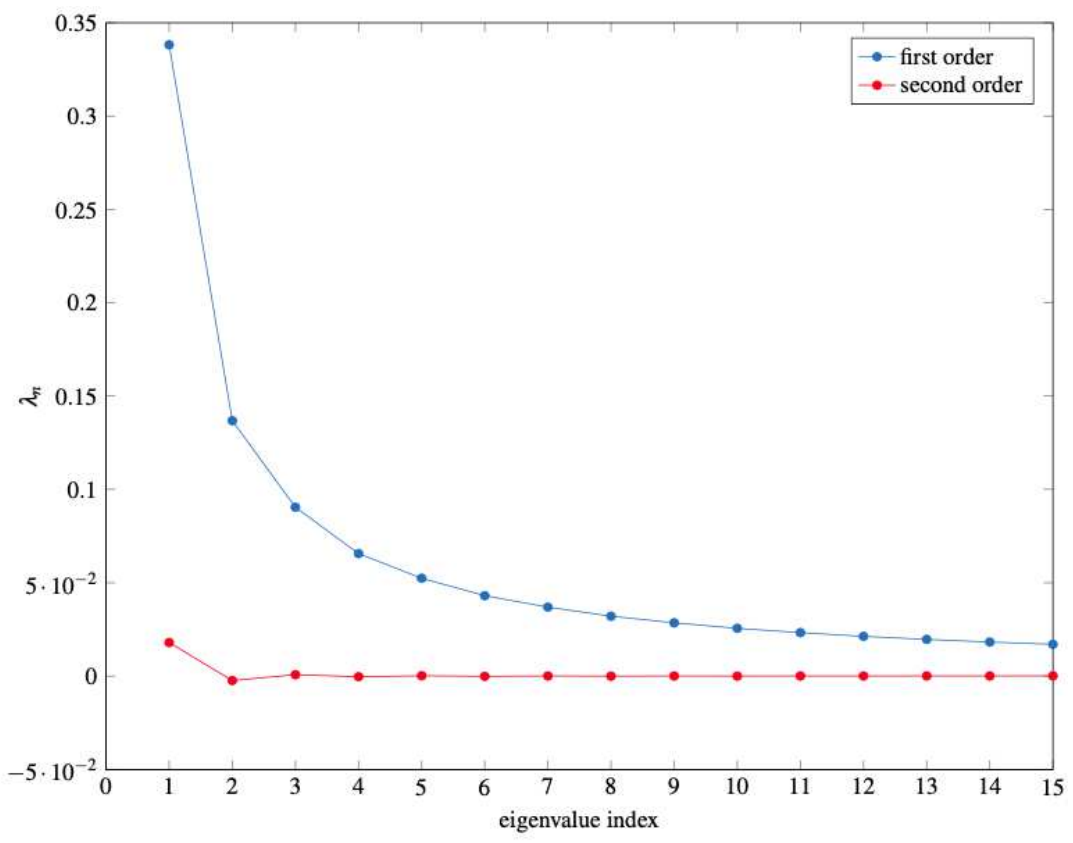


Figure 1. The relative error $\widehat{\lambda}_{n} / \widetilde{\lambda}_{n}^{(i)}-1$ versus $n$ for $\alpha=3 / 4$, where $\widehat{\lambda}_{n}$ is a high precision numerical approximation of the eigenvalues $\lambda_{n}$ and

$$
\widetilde{\lambda}_{n}^{(1)}=(\pi n)^{2 \alpha} \quad \text { and } \quad \widetilde{\lambda}_{n}^{(2)}=\left(\pi n+\frac{\pi}{2}\left(1-\frac{1}{\alpha}\right)\right)^{2 \alpha}
$$

are the first and second order asymptotic approximations.

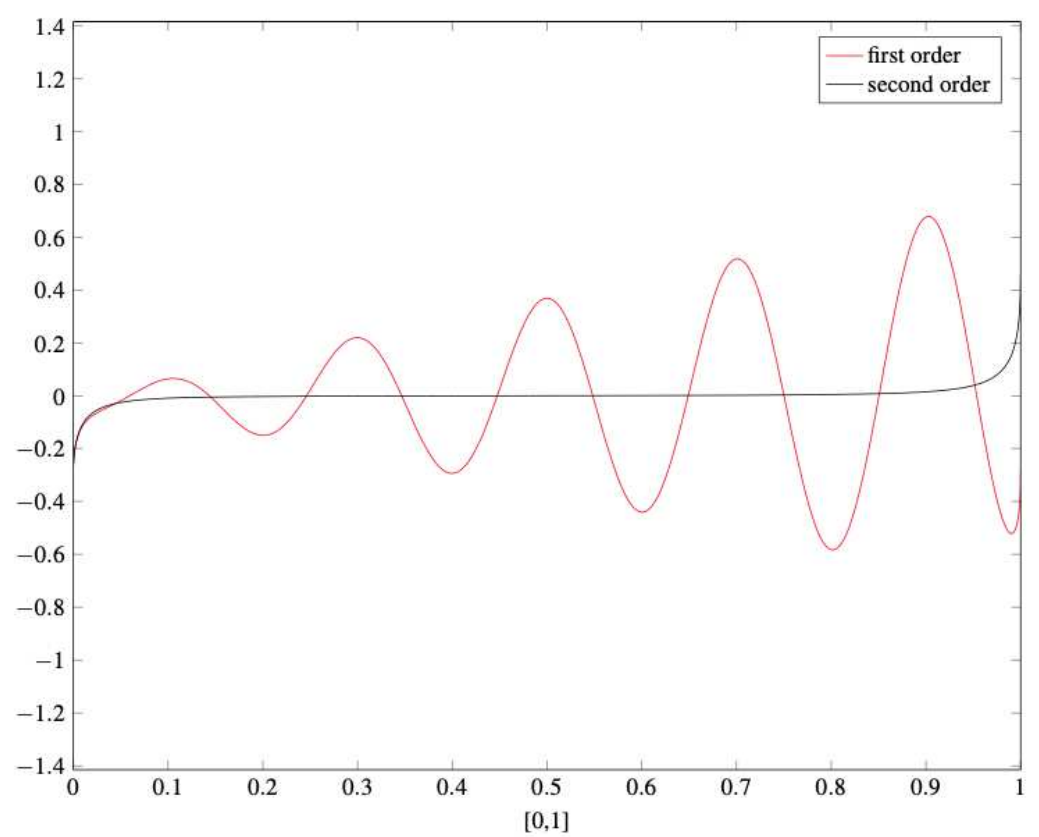

Figure 2. The error $\widehat{f}_{n}(x)-\widetilde{f}_{n}^{(i)}(x)$ versus $x \in[0,1]$ for $\alpha=3 / 4$ and $n=10$, where $\widehat{f}_{n}$ is a high precision numerical approximation of the eigenfunction $f_{n}$ and

$$
\widetilde{f}_{n}^{(i)}=\sqrt{2} \sin \left(\widehat{\rho}_{n}^{(i)} x+\frac{\pi}{4}(1-\alpha)\right), \quad i=1,2,
$$

with $\widehat{\rho}_{n}^{(i)}=\left(\widehat{\lambda}^{(i)}\right)^{1 /(2 \alpha)}$, are the first and second order approximations for the oscillating part of the eigenfunctions. The effect of truncated boundary layer terms is clearly visible for the second order approximation.

c) Problem $(\mathrm{P})$ is closely related to a slightly different problem

$$
\begin{aligned}
& { }^{c} D_{1-}^{\alpha}{ }^{c} D_{0+}^{\alpha} u(x)=\lambda u(x), \quad x \in[0,1], \\
& u(0)=0,{ }^{c} D_{0+}^{\alpha} u(1)=0,
\end{aligned}
$$


with the left and right Caputo derivatives

$$
\begin{aligned}
& { }^{c} D_{0+}^{\alpha} f(x):=D_{0+}^{\alpha}(f(x)-f(0)), \\
& { }^{c} D_{1-}^{\alpha} f(x):=D_{1-}^{\alpha}(f(x)-f(1)) .
\end{aligned}
$$

Reduction to the integral operators as in [16], [19] reveals that $\left(\mathrm{P}^{\prime}\right)$ with $\alpha \in(0,1)$ is equivalent to the problem

$$
\int_{0}^{1} K(x, y) f(y) d y=\lambda^{-1} f(x), \quad x \in[0,1],
$$

with the kernel

$$
K(x, y)=\frac{1}{\Gamma(\alpha)^{2}} \int_{0}^{x \wedge y}(x-y)^{\alpha-1}(y-t)^{\alpha-1} d t
$$

while problem $(\mathrm{P})$ is equivalent to

$$
\int_{0}^{1}\left(K(x, y)-\frac{K(x, 1) K(1, y)}{K(1,1)}\right) f(y) d y=\lambda^{-1} f(x), \quad x \in[0,1],
$$

with $K(x, y)$ as in (2.4).

Our approach applies to problem $\left(\mathrm{P}^{\prime}\right)$ with minor adjustments and it can be seen that the assertion of Theorem 2.1 remains valid, this time for $\alpha \in(0,1)$, with

$$
\rho_{n}=\pi n-\frac{\pi}{2}+O\left(n^{-1}\right),
$$

and different but still explicit functions $\Upsilon_{j}(t)$. Curiously, in this case the solutions can be shown to have very particular value at the right endpoint,

$$
\left|f_{n}(1)\right|=\sqrt{2 \alpha}
$$

at least for all sufficiently large $n$.

In the theory of stochastic processes, kernel (2.4) with $\alpha \in\left(\frac{1}{2}, 1\right)$ is the covariance function of the so called Riemann-Liouville process, one of the two most common fractional generalisations of the standard Brownian motion. The kernel in (2.5) with $\alpha \in\left(\frac{1}{2}, 1\right)$ is the covariance function of the corresponding bridge, obtained by conditioning the Riemann-Liouville process to vanish at $x=1$.

For $\alpha \in\left(0, \frac{1}{2}\right]$ the covariance function (2.4) is still well defined, but it remains continuous only off diagonal, where it has a weak singularity. Such covariance function does not correspond to a stochastic process in the usual sense, as it cannot have continuous paths. Instead it can be interpreted as covariance of the fractional noise process, a formal derivative of the Riemann-Liouville process. This can be made precise by e.g., considering stochastic integrals, see 7]. Obviously, the bridge kernel as in (2.5) can no longer be defined in this case. In fact, our analysis implies that problem $(\mathrm{P})$ with $\alpha \in\left(0, \frac{1}{2}\right)$ cannot have more than finitely many continuous solutions. 


\section{Proof of Theorem 2.1}

The proof is based on the approach to analysis of integral equations with weakly singular kernels, introduced in [29]. In its original form this method applies to operators with difference kernels, see also [26], 27]. Recently it was extended to various forms of integrated difference kernels, which allowed to compute the exact spectral asymptotics of covariance operators for a number of stochastic processes, including the fractional Brownian motion, 7], 6], [14, [24.

While inversion of the fractional derivatives in problem $(\mathrm{P})$ still yields an integral operator, its kernel does not seem to have any simple difference structure. The principal contribution of this paper is generalisation of the method to the integro-differential operators of the fractional types, which makes it potentially applicable to corresponding Sturm-Liouville problems. To avoid repetitions and focus only on the new elements of the proof, we will omit calculations, which can be found elsewhere and provide with exact references.

3.1. Conventions and notations. The proof uses some standard notations and classical tools from complex analysis. Unless stated otherwise, we will use the conventional principal branches for multivalued functions. Often we will encounter functions, which are holomorphic on the complex plane, cut along the real (semi-) axis with a finite jump discontinuity across the cut. For such a sectionally holomorphic function $\Psi(z)$, we will use the limit notations

$$
\Psi^{+}(t)=\lim _{\operatorname{Im}(z)>0, z \rightarrow t} \Psi(z) \quad \text { and } \quad \Psi^{-}(t)=\lim _{\operatorname{Im}(z)<0, z \rightarrow t} \Psi(z), \quad t \in \mathbb{R} .
$$

Finding a function $\Psi(z)$, sectionally holomorphic on $\mathbb{C} \backslash \mathbb{R}_{+}$and satisfying the boundary condition

$$
\Psi^{+}(t)-\Psi^{-}(t)=g(t), \quad t \in \mathbb{R}_{+},
$$

where $g(\cdot)$ is a given Hölder function on $\mathbb{R}_{+} \cup\{\infty\}$, is known as the Hilbert boundary value problem. The solution is given by the Sokhotski-Plemelj formula

$$
\Psi(z)=\frac{1}{2 \pi i} \int_{0}^{\infty} \frac{g(t)}{t-z} d t+P(z), \quad z \in \mathbb{C} \backslash \mathbb{R}_{+},
$$

where $P(\cdot)$ is a polynomial, which matches the growth of $\Psi(\cdot)$ at infinity. A comprehensive account of the related theory can be found in [10].

3.2. A brief preview. In a nutshell the main idea of the method is to reduce eigenproblem $(\mathrm{P})$ to an equivalent integro-algebraic system of equations, more tractable for asymptotic analysis. In Lemma 3.1] below we show that the Laplace transform 


$$
\widehat{f}(z)=\int_{0}^{1} e^{-z x} f(x) d x, \quad z \in \mathbb{C},
$$

of a solution $f$ to problem $(\mathrm{P})$ satisfies the representation

$$
\widehat{f}(z)=-\frac{c_{f}}{\lambda} e^{-z}-\frac{\Phi_{0}(z)+e^{-z} \Phi_{1}(-z)}{\Lambda(z)}, \quad z \in \mathbb{C},
$$

where functions $\Phi_{0}(z)$ and $\Phi_{1}(z)$ are sectionally holomorphic on $\mathbb{C} \backslash \mathbb{R}_{+}$ and $c_{f}$ is a functional of $f$, constant in $z$. The function $\Lambda(z)$ is defined by a closed form expression, which vanishes only at a pair of purely imaginary conjugate zeros and is sectionally holomorphic on the cut plane $\mathbb{C} \backslash \mathbb{R}$.

Since a priori the Laplace transform $\widehat{f}(z)$ is an entire function, all singularities in (3.2) must be removable. Removing discontinuity along the real line yields representations of $\Phi_{0}(z)$ and $\Phi_{1}(z)$ in terms of solutions to an auxiliary system of integral equations on $\mathbb{R}_{+}$, see Lemma 3.4. Removal of the poles produces an algebraic condition, which binds together values of these functions at certain points in the complex plane. This integroalgebraic system of equations, described in Lemma 3.5, is shoen to have countably many solutions, whose asymptotic analysis leads to the expressions claimed in Theorem 2.1 .

Thereby problem $(\mathrm{P})$ reduces to finding a pair of functions, sectionally holomorphic on $\mathbb{C} \backslash \mathbb{R}_{+}$with a given jump discontinuity on $\mathbb{R}_{+}$and specific growth near the origin and at infinity. Remarkably, this seemingly more complicated problem turns out to be amenable to asymptotic analysis.

3.3. The Laplace transform. Our starting point is the following representation for the Laplace transform.

Lemma 3.1. Let $(\lambda, f)$ be a solution to $(\mathrm{P})$. The Laplace transform (3.1) of $f$ satisfies the representation

$$
\widehat{f}(z)=-\frac{c_{f}}{\lambda} e^{-z}-\frac{\Phi_{0}(z)+e^{-z} \Phi_{1}(-z)}{\Lambda(z)}, \quad z \in \mathbb{C},
$$

where $c_{f}=\lim _{x \rightarrow 1} I_{1-}^{1-\alpha} D_{0+}^{\alpha} f(x)$,

$$
\Lambda(z)=\frac{\pi}{\sin (\alpha \pi)}\left(z^{\alpha}-\lambda(-z)^{-\alpha}\right)
$$

and functions $\Phi_{0}(z)$ and $\Phi_{1}(z)$, defined in (3.12) below, are sectionally holomorphic on $\mathbb{C} \backslash \mathbb{R}_{+}$and satisfy the growth estimates

$$
\Phi_{0}(z)=O\left(z^{-\alpha}\right) \quad \text { and } \quad \Phi_{1}(z)=O\left(z^{\alpha}\right), \quad z \rightarrow \infty,
$$

and

$$
\Phi_{0}(z)=O\left(z^{-\alpha}\right) \quad \text { and } \quad \Phi_{1}(z)=O(1), \quad z \rightarrow 0 .
$$


P r o o f. Applying the left Riemann-Liouville integral

$$
\left(I_{1-}^{\alpha} f\right)(x)=\frac{1}{\Gamma(\alpha)} \int_{x}^{1}(t-x)^{\alpha-1} f(t) d t
$$

to both sides of the equation in $(\mathrm{P})$ and using the composition rule $[13$, Lemma 2.6], we can write

$$
D_{0+}^{\alpha} f(x)-\frac{c_{f}}{\Gamma(\alpha)}(1-x)^{\alpha-1}=\lambda I_{1-}^{\alpha} f(x) .
$$

Following [24], define the function

then

$$
\psi(x)=\int_{x}^{1} f(y) d y+\frac{c_{f}}{\lambda},
$$

$$
\begin{aligned}
& \lambda I_{1-}^{\alpha} f(x)+\frac{c_{f}}{\Gamma(\alpha)}(1-x)^{\alpha-1} \\
& =-\frac{\lambda}{\Gamma(\alpha)} \int_{x}^{1} \psi^{\prime}(y)(y-x)^{\alpha-1} d y+\frac{c_{f}}{\Gamma(\alpha)}(1-x)^{\alpha-1} \\
& =\frac{\lambda}{\Gamma(\alpha)} \frac{1}{\alpha} \frac{d}{d x} \int_{x}^{1} \psi^{\prime}(y)(y-x)^{\alpha} d y+\frac{c_{f}}{\Gamma(\alpha)}(1-x)^{\alpha-1} \\
& =\frac{\lambda \psi(1)}{\Gamma(\alpha) \alpha} \frac{d}{d x}(1-x)^{\alpha}-\frac{\lambda}{\Gamma(\alpha)} \frac{d}{d x} \int_{x}^{1} \psi(y)(y-x)^{\alpha-1} d y+\frac{c_{f}}{\Gamma(\alpha)}(1-x)^{\alpha-1} \\
& =-\frac{\lambda}{\Gamma(\alpha)} \frac{d}{d x} \int_{x}^{1} \psi(y)(y-x)^{\alpha-1} d y
\end{aligned}
$$

Thus problem $(\mathrm{P})$ takes the equivalent form

$$
\begin{aligned}
& \frac{1}{\Gamma(1-\alpha)} \frac{d}{d x} \int_{0}^{x} \psi^{\prime}(y)(x-y)^{-\alpha} d y=\frac{\lambda}{\Gamma(\alpha)} \frac{d}{d x} \int_{x}^{1} \psi(y)(y-x)^{\alpha-1} d y, \\
& \psi^{\prime}(0)=\psi^{\prime}(1)=0 .
\end{aligned}
$$

Using the identity

$$
s^{-r}=\frac{1}{\Gamma(r)} \int_{0}^{\infty} t^{r-1} e^{-s t} d t, \quad r>0, s>0,
$$

the equation in (3.8) can be written as

where we denoted

$$
v_{0}^{\prime}(x)=\lambda u_{0}^{\prime}(x)
$$

$$
u_{0}(x)=\int_{0}^{\infty} t^{-\alpha} u(x, t) d t, \quad u(x, t)=\int_{x}^{1} \psi(y) e^{-t(y-x)} d y
$$

and

$$
v_{0}(x)=\int_{0}^{\infty} t^{\alpha-1} v(x, t) d t, \quad v(x, t)=\int_{0}^{x} \psi^{\prime}(y) e^{-t(x-y)} d y .
$$

Differentiating with respect to $x$ shows that $u(x, t)$ solves the differential equation 


$$
\begin{aligned}
u^{\prime}(x, t) & =t u(x, t)-\psi(x), \\
u(1, t) & =0 .
\end{aligned}
$$

Applying the Laplace transform we get

$$
\widehat{u}^{\prime}(z, t)=t \widehat{u}(z, t)-\widehat{\psi}(z) .
$$

On the other hand, $\widehat{u}^{\prime}(z, t)=-u(0, t)+z \widehat{u}(z, t)$, and therefore

$$
\widehat{u}(z, t)=\frac{\widehat{\psi}(z)-u(0, t)}{t-z} .
$$

It follows that

$$
\widehat{u}_{0}(z)=\widehat{\psi}(z) M_{u}(z)-\int_{0}^{\infty} \frac{t^{-\alpha}}{t-z} u(0, t) d t
$$

where

$$
M_{u}(z):=\int_{0}^{\infty} \frac{t^{-\alpha}}{t-z} d t=\frac{\pi}{\sin (\alpha \pi)}(-z)^{-\alpha} .
$$

The last equality holds due to the identity

$$
\int_{0}^{\infty} \frac{t^{-\beta}}{t+z} d t=\frac{\pi}{\sin (\pi \beta)} z^{-\beta}, \quad \beta \in(0,1), \arg (z) \in[-\pi, \pi) .
$$

Similarly,

$$
\begin{aligned}
v^{\prime}(x, t) & =-t v(x, t)+\psi^{\prime}(x), \\
v(0, t) & =0,
\end{aligned}
$$

and, since $\widehat{v}^{\prime}(z, t)=v(1, t) e^{-z}+z \widehat{v}(z, t)$, we get

$$
\widehat{v}(z, t)=\frac{\widehat{\psi}^{\prime}(z)-v(1, t) e^{-z}}{t+z}=\frac{\psi(1)-v(1, t)}{t+z} e^{-z}+\frac{z \widehat{\psi}(z)-\psi(0)}{t+z},
$$

and consequently,

$$
\begin{aligned}
\widehat{v}_{0}(z)= & e^{-z} \psi(1) M_{v}(-z)-e^{-z} \int_{0}^{\infty} \frac{t^{\alpha-1}}{t+z} v(1, t) d t \\
& +(z \widehat{\psi}(z)-\psi(0)) M_{v}(-z)
\end{aligned}
$$

where we defined

$$
M_{v}(z)=\int_{0}^{\infty} \frac{t^{\alpha-1}}{t-z} d t=\frac{\pi}{\sin (\alpha \pi)}(-z)^{\alpha-1} .
$$

In the Laplace domain, the equation (3.9) is equivalent to

$$
z\left(\widehat{v}_{0}(z)-\lambda \widehat{u}_{0}(z)\right)=c\left(1-e^{-z}\right),
$$

where $c:=v_{0}(1)=-\lambda u_{0}(0)$. Plugging (3.10) and (3.11) into this equation and rearranging we arrive at 


$$
z \widehat{\psi}(z)-\psi(0)=\frac{\Phi_{0}(z)+e^{-z} \Phi_{1}(-z)}{\Lambda(z)}
$$

where

and

$$
\begin{aligned}
& \Phi_{0}(z)=\psi(0) \lambda M_{u}(z)-\lambda \int_{0}^{\infty} \frac{t^{1-\alpha}}{t-z} u(0, t) d t \\
& \Phi_{1}(z)=\psi(1) z M_{v}(z)-\int_{0}^{\infty} \frac{t^{\alpha}}{t-z} v(1, t) d t
\end{aligned}
$$

$$
\Lambda(z)=z M_{v}(-z)-\lambda M_{u}(z)=\frac{\pi}{\sin (\alpha \pi)}\left(z^{\alpha}-\lambda(-z)^{-\alpha}\right) .
$$

Representation (3.3) follows from (3.12) since $z \widehat{\psi}(z)-\psi(0)=-\widehat{f}(z)-$ $e^{-z} \psi(1)$. The growth estimates (3.6) and (3.5) are verified by standard calculations.

The function $\Lambda(z)$ largely determines the structure of the problem. The following lemma summarises some of its key properties, relevant to further analysis.

\section{LEMMA 3.2.}

a) The function $\Lambda(z)$ in (3.4) is sectionally holomorphic on $\mathbb{C} \backslash \mathbb{R}$ and discontinuous across the real line with the limits

$$
\Lambda^{ \pm}(t)=\frac{\pi}{\sin (\alpha \pi)} \begin{cases}t^{\alpha}-\lambda e^{ \pm \alpha \pi i} t^{-\alpha}, & t>0, \\ e^{ \pm \alpha \pi i}|t|^{\alpha}-\lambda|t|^{-\alpha}, & t<0,\end{cases}
$$

which satisfy the symmetries

$$
\begin{aligned}
& \Lambda^{+}(t)=\overline{\Lambda^{-}(t)} \\
& \Lambda^{+}(t)=e^{\alpha \pi i} \Lambda^{-}(-t), \\
& \frac{\Lambda^{+}(t)}{\Lambda^{-}(t)}=e^{2 \alpha \pi i} \frac{\Lambda^{-}(-t)}{\Lambda^{+}(-t)} .
\end{aligned}
$$

b) As $t$ varies from 0 to $\infty$, the angle $\theta(t)=\arg \left\{\Lambda^{+}(t)\right\}$ increases continuously from $\theta(0+)=(\alpha-1) \pi<0$ to $\theta(\infty)=0$ and

$$
|\theta(t)|=O\left(t^{-2 \alpha}\right), \quad \text { as } \rightarrow \infty .
$$

c) $\Lambda(z)$ vanishes only at simple zeros $\pm z_{0}= \pm i \rho$ with

$$
\rho=\lambda^{1 /(2 \alpha)},
$$

and the function $\theta_{0}(t):=\theta(\rho t)$ does not depend on $\rho$.

P r o o f. All the claims are verified by direct calculations. 
3.4. Removal of singularities. Since the integration in (3.1) is over a finite interval, $\widehat{f}(z)$ is an entire function and therefore all singularities in (3.3) must be removable. The discontinuity is removed by equating the limits in the upper and lower half planes,

$$
\begin{aligned}
& \frac{1}{\Lambda^{+}(t)}\left(e^{-t} \Phi_{1}(-t)+\Phi_{0}^{+}(t)\right)=\frac{1}{\Lambda^{-}(t)}\left(e^{-t} \Phi_{1}(-t)+\Phi_{0}^{-}(t)\right), \quad t \in \mathbb{R}_{+}, \\
& \frac{1}{\Lambda^{+}(t)}\left(e^{-t} \Phi_{1}^{-}(-t)+\Phi_{0}(t)\right)=\frac{1}{\Lambda^{-}(t)}\left(e^{-t} \Phi_{1}^{+}(-t)+\Phi_{0}(t)\right), \quad t \in \mathbb{R}_{-},
\end{aligned}
$$

which, in view of (3.14), can be written as

$$
\begin{aligned}
& \Phi_{0}^{+}(t)-\frac{\Lambda^{+}(t)}{\Lambda^{-}(t)} \Phi_{0}^{-}(t)=e^{-t} \Phi_{1}(-t)\left(\frac{\Lambda^{+}(t)}{\Lambda^{-}(t)}-1\right), \\
& \Phi_{1}^{+}(t)-e^{-2 \alpha \pi i} \frac{\Lambda^{+}(t)}{\Lambda^{-}(t)} \Phi_{1}^{-}(t)=e^{-t} \Phi_{0}(-t)\left(1-e^{-2 \alpha \pi i} \frac{\Lambda^{+}(t)}{\Lambda^{-}(t)}\right), \quad t \in \mathbb{R}_{+} .
\end{aligned}
$$

Removal of the poles in (3.3) implies

$$
\Phi_{0}\left(z_{0}\right)+\Phi_{1}\left(-z_{0}\right) e^{-z_{0}}=0
$$

since the zeros of $\Lambda(z)$ are purely imaginary and conjugate, and $\overline{\Phi_{j}(z)}=$ $\Phi_{j}(\bar{z})$ by definition (3.13).

3.5. Auxiliary integro-algebraic system. The next step is to show that any pair of functions $\Phi_{0}(z)$ and $\Phi_{1}(z)$, which are sectionally holomorphic on the cut plane $\mathbb{C} \backslash \mathbb{R}_{+}$, satisfy boundary conditions (3.15) and growth estimates (3.6)-(3.5), solve a certain auxiliary system of integral equations. Along with condition (3.16) these equations form an integro-algebraic system, whose solutions correspond to solutions of the spectral problem $(\mathbb{P})$ under consideration.

To this end we will use the classical technique of solving the Hilbert boundary value problems, see e.g. [10]. Consider the function

$$
X_{c}(z)=\exp \left(\frac{1}{\pi} \int_{0}^{\infty} \frac{\theta(t)}{t-z} d t\right), \quad z \in \mathbb{C} \backslash \mathbb{R}_{+},
$$

where $\theta(t)$ was introduced in Lemma 3.2, By the Sokhotski-Plemelj theorem, $X_{c}(z)$ is sectionally holomorphic on $\mathbb{C} \backslash \mathbb{R}_{+}$and its limits satisfy

$$
\frac{X_{c}^{+}(t)}{X_{c}^{-}(t)}=e^{2 i \theta(t)}=\frac{\Lambda^{+}(t)}{\Lambda^{-}(t)}, \quad t \in \mathbb{R}_{+}
$$

where the last equality holds by (3.14). Some useful properties of this function are gathered in the following lemma. 
Lemma 3.3. The function $X_{c 0}(z):=X_{c}(\rho z)$ does not depend on $\rho$, satisfies the growth estimates

$$
X_{c 0}(z)= \begin{cases}O\left(z^{1-\alpha}\right), & \text { as } z \rightarrow 0 \\ 1-b_{\alpha} z^{-1}+O\left(z^{-2 \alpha}\right), & \text { as } z \rightarrow \infty\end{cases}
$$

with $b_{\alpha}=\cot \left(\frac{\pi}{2 \alpha}\right)$, and has the following explicit value at $z=i$,

$$
X_{c 0}(i)=\sqrt{\alpha} \exp \left(-\frac{\pi}{4}(1-\alpha) i\right) .
$$

P r o o f. Evaluating (3.17) at $\rho z$ and changing integration variable accordingly gives

$$
X_{c 0}(z)=\exp \left(\frac{1}{\pi} \int_{0}^{\infty} \frac{\theta_{0}(t)}{t-z} d t\right)
$$

where $\theta_{0}(t)$ does not depend on $\rho$, see Lemma 3.2 (c). The estimate near the origin in (3.19) is obtained by means of integration by parts,

$$
X_{c 0}(z)=(-z)^{-\theta_{0}(0+) / \pi} \exp \left(\frac{1}{\pi} \int_{0}^{\infty} \theta_{0}^{\prime}(t) \log (t-z) d t\right)=O\left(z^{1-\alpha}\right), z \rightarrow 0,
$$

since $\theta_{0}(0+)=(\alpha-1) \pi$, see Lemma 3.2 (b). The estimate at infinity holds since

$$
\begin{gathered}
X_{c 0}(z)=\exp \left(-z^{-1} \frac{1}{\pi} \int_{0}^{\infty} \theta_{0}(t) d t+z^{-1} \frac{1}{\pi} \int_{0}^{\infty} \frac{t \theta_{0}(t)}{t-z} d t\right) \\
=1-z^{-1} b_{\alpha}+O\left(z^{-2 \alpha}\right), \quad z \rightarrow \infty
\end{gathered}
$$

where

$$
b_{\alpha}:=\frac{1}{\pi} \int_{0}^{\infty} \theta_{0}(t) d t
$$

The exact value of the latter integral and the constant in (3.20) are found by direct calculation, which use the explicit expressions for $\Lambda^{ \pm}(t)$ from Lemma 3.2, similarly to [7, Lemma 5.5 ].

Define $X(z):=z^{-1} X_{c}(z)$ and $Y(z):=(-z)^{\alpha-1} X_{c}(z)$. These functions are sectionally holomorphic on $\mathbb{C} \backslash \mathbb{R}_{+}$and, in view of (3.18), satisfy boundary conditions

$$
\begin{aligned}
& \frac{X^{+}(t)}{X^{-}(t)}=\frac{\Lambda^{+}(t)}{\Lambda^{-}(t)}, \\
& \frac{Y^{+}(t)}{Y^{-}(t)}=e^{-2 \alpha \pi i} \frac{\Lambda^{+}(t)}{\Lambda^{-}(t)}, \quad t \in \mathbb{R}_{+} .
\end{aligned}
$$


Plugging these formulas into equations (3.15) and rearranging gives

$$
\begin{aligned}
& \frac{\Phi_{0}^{+}(t)}{X^{+}(t)}-\frac{\Phi_{0}^{-}(t)}{X^{-}(t)}=e^{-t} Y(-t)\left(\frac{1}{X^{-}(t)}-\frac{1}{X^{+}(t)}\right) \frac{\Phi_{1}(-t)}{Y(-t)}, \quad t \in \mathbb{R}_{+} . \\
& \frac{\Phi_{1}^{+}(t)}{Y^{+}(t)}-\frac{\Phi_{1}^{-}(t)}{Y^{-}(t)}=e^{-t} X(-t)\left(\frac{1}{Y^{+}(t)}-\frac{1}{Y^{-}(t)}\right) \frac{\Phi_{0}(-t)}{X(-t)},
\end{aligned}
$$

This shows that the functions

$$
\Psi_{0}(z)=\frac{\Phi_{0}(z)}{X(z)} \quad \text { and } \quad \Psi_{1}(z)=\frac{\Phi_{1}(z)}{Y(z)}
$$

are sectionally holomorphic on $\mathbb{C} \backslash \mathbb{R}_{+}$with the boundary conditions

$$
\begin{aligned}
& \Psi_{0}^{+}(t)-\Psi_{0}^{-}(t)=2 i e^{-t} g(t) \Psi_{1}(-t), \\
& \Psi_{1}^{+}(t)-\Psi_{1}^{-}(t)=2 i e^{-t} h(t) \Psi_{0}(-t),
\end{aligned} \quad t \in \mathbb{R}_{+},
$$

where

$$
\begin{aligned}
g(t) & :=\frac{1}{2 i} Y(-t)\left(\frac{1}{X^{-}(t)}-\frac{1}{X^{+}(t)}\right), \\
h(t) & :=\frac{1}{2 i} X(-t)\left(\frac{1}{Y^{+}(t)}-\frac{1}{Y^{-}(t)}\right) .
\end{aligned}
$$

These two functions turn out to be real valued and satisfy the scaling properties

$$
\begin{aligned}
& g(\rho t)=\rho^{\alpha} t^{\alpha} \sin \left(\theta_{0}(t)\right) \exp \left(-\frac{2 t}{\pi} \int_{0}^{\infty} \frac{\theta_{0}(\tau)}{\tau^{2}-t^{2}} d \tau\right)=: \rho^{\alpha} g_{0}(t), \\
& h(\rho t)=-\rho^{-\alpha} t^{-\alpha} \sin \left(\theta_{0}(t)-\alpha \pi\right) \exp \left(-\frac{2 t}{\pi} f_{0}^{\infty} \frac{\theta_{0}(\tau)}{\tau^{2}-t^{2}} d \tau\right)=: \rho^{-\alpha} h_{0}(t),
\end{aligned}
$$

where the integral is in the sense of Cauchy's principal value and $g_{0}(\cdot)$ and $h_{0}(\cdot)$ do not depend on $\rho$.

In view of estimates (3.6) and (3.19), both $\Psi_{0}(z)$ and $\Psi_{1}(z)$ are bounded in the vicinity of zero and therefore, due to the Sokhotski-Plemelj theorem, satisfy

$$
\begin{aligned}
& \Psi_{0}(z)=\frac{1}{\pi} \int_{0}^{\infty} \frac{e^{-\tau} g(\tau)}{\tau-z} \Psi_{1}(-\tau) d \tau+P_{0}(z), \\
& \Psi_{1}(z)=\frac{1}{\pi} \int_{0}^{\infty} \frac{e^{-\tau} h(\tau)}{\tau-z} \Psi_{0}(-\tau) d \tau+P_{1}(z),
\end{aligned}
$$

where $P_{j}(z)$ 's are polynomials, whose growth at infinity matches that of $\Psi_{j}(z)$ 's. 
Let us estimate the growth of $\Psi_{1}(z)$ as $z \rightarrow \infty$. To this end note that the integral term of $\Phi_{1}(z)$ in (3.13) satisfies

$$
\begin{aligned}
& z^{1-\alpha} \int_{0}^{\infty} \frac{t^{\alpha}}{t-z} v(1, t) d t=z^{1-\alpha}|z|^{\alpha} \int_{0}^{\infty} \frac{t^{\alpha}}{t-z /|z|} v(1, t|z|) d t \\
& =(z /|z|)^{1-\alpha} \int_{0}^{\infty} \frac{t^{\alpha-1}}{t-z /|z|} \int_{0}^{1} \psi^{\prime}(y)|z| t e^{-|z| t(1-y)} d y d t \stackrel{|z| \rightarrow \infty}{\longrightarrow} 0,
\end{aligned}
$$

where the limit holds, since the inner integral converges to $\psi^{\prime}(1)=0$. Consequently, as $z \rightarrow \infty$,

$$
\begin{aligned}
\Psi_{1}(z)= & \frac{\Phi_{1}(z)}{Y(z)}=\psi(1) \frac{\pi}{\sin (\alpha \pi)} \frac{z}{X_{c}(z)}(1+o(1)) \\
& =\psi(1) \frac{\pi}{\sin (\alpha \pi)}\left(\rho b_{\alpha}+z\right)(1+o(1)), \quad z \rightarrow \infty .
\end{aligned}
$$

Similar calculations show that $\Psi_{0}(z)=O(1)$ as $z \rightarrow \infty$. In view of these estimates (3.22) takes the form

$$
\begin{array}{ll}
\Psi_{0}(z)=\frac{1}{\pi} \int_{0}^{\infty} \frac{e^{-\tau} g(\tau)}{\tau-z} \Psi_{1}(-\tau) d \tau+c_{0}, & z \in \mathbb{C} \backslash \mathbb{R}_{+}, \\
\Psi_{1}(z)=\frac{1}{\pi} \int_{0}^{\infty} \frac{e^{-\tau} h(\tau)}{\tau-z} \Psi_{0}(-\tau) d \tau+c_{1}\left(\rho b_{\alpha}+z\right), &
\end{array}
$$

where $c_{0}$ and $c_{1}$ are real constants,

$$
c_{1}=\psi(1) \frac{\pi}{\sin (\alpha \pi)}=\frac{c_{f}}{\lambda} \frac{\pi}{\sin (\alpha \pi)} .
$$

In particular, for $z=-t$ we obtain the system of integral equations for $\Psi_{0}(-t)$ and $\Psi_{1}(-t)$,

$$
\begin{aligned}
& \Psi_{0}(-t)=\frac{1}{\pi} \int_{0}^{\infty} \frac{e^{-\tau} g(\tau)}{\tau+t} \Psi_{1}(-\tau) d \tau+c_{0}, \\
& \Psi_{1}(-t)=\frac{1}{\pi} \int_{0}^{\infty} \frac{e^{-\tau} h(\tau)}{\tau+t} \Psi_{0}(-\tau) d \tau+c_{1}\left(\rho b_{\alpha}-t\right),
\end{aligned} \quad t \in \mathbb{R}_{+} .
$$

In view of the growth estimates for $\Psi_{0}(z)$ and $\Psi_{1}(z)$, the integrals in the right hand side define functions in $L_{2}\left(\mathbb{R}_{+}, \mathbb{R}\right)$. Finally, condition (3.16) reads

$$
\Psi_{0}(i \rho) X(i \rho)+\Psi_{1}(-i \rho) Y(-i \rho) e^{-i \rho}=0 .
$$

Thus we arrive at the following result.

Lemma 3.4. Let $(\lambda, f)$ be a solution to $(\mathrm{P})$ and define $\Phi_{0}(z)$ and $\Phi_{1}(z)$ by (3.13). Then the functions $\Psi_{0}(z)$ and $\Psi_{1}(z)$, defined in (3.21), satisfy (3.25) and solve the integral equations (3.24), where $\rho=\lambda^{1 /(2 \alpha)}$ and coefficients $c_{0}$ and $c_{1}$ are determined by (3.23) and $c_{0}=\lim _{z \rightarrow \infty} \Psi_{0}(z)$. 
3.6. Structure of the auxiliary system. Define the operator

$$
A f(t):=\frac{1}{\pi} \int_{0}^{\infty} \frac{e^{-\rho \tau}}{\tau+t}\left(\begin{array}{cc}
0 & g_{0}(\tau) \\
h_{0}(\tau) & 0
\end{array}\right) f(\tau) d \tau
$$

which acts on functions $f: \mathbb{R}_{+} \mapsto \mathbb{R}^{2}$, and consider the systems of integral equations

$$
\begin{aligned}
& p(t)=A p(t)+\left(\begin{array}{l}
1 \\
0
\end{array}\right), \\
& q(t)=A q(t)+\left(\begin{array}{l}
0 \\
1
\end{array}\right), \quad t \in \mathbb{R}_{+} . \\
& r(t)=A r(t)+\left(\begin{array}{l}
0 \\
t
\end{array}\right),
\end{aligned}
$$

By changing variables, equations (3.24) can be written as

$$
\begin{array}{ll}
\Psi_{0}(-\rho t) & =\rho^{\alpha} \frac{1}{\pi} \int_{0}^{\infty} \frac{e^{-\rho \tau} g_{0}(\tau)}{\tau+t} \Psi_{1}(-\rho \tau) d \tau+c_{0}, \\
\Psi_{1}(-\rho t) & =\rho^{-\alpha} \frac{1}{\pi} \int_{0}^{\infty} \frac{e^{-\rho \tau} h_{0}(\tau)}{\tau+t} \Psi_{0}(-\rho \tau) d \tau+c_{1}\left(\rho b_{\alpha}-\rho t\right),
\end{array} \quad t \in \mathbb{R}_{+},
$$

and therefore, by linearity,

$$
\left(\begin{array}{c}
\rho^{-\alpha} \Psi_{0}(\rho z) \\
\Psi_{1}(\rho z)
\end{array}\right)=\rho^{-\alpha} c_{0} p(-z)+c_{1} \rho b_{\alpha} q(-z)-c_{1} \rho r(-z),
$$

where solutions to (3.27) are extended to $\mathbb{C} \backslash \mathbb{R}$ - by analyticity. Plugging these expressions into (3.25) gives

$$
c_{0} \xi(\rho)+c_{1} \eta(\rho)=0,
$$

where we denoted

$$
\begin{aligned}
& \xi(\rho):=X(\rho i) p_{1}(-i)+\rho^{-\alpha} e^{-\rho i} Y(-\rho i) p_{2}(i), \\
& \eta(\rho):=X(\rho i) \rho^{\alpha}\left(\rho b_{\alpha} q_{1}(-i)-\rho r_{1}(-i)\right)+e^{-\rho i} Y(-\rho i)\left(\rho b_{\alpha} q_{2}(i)-\rho r_{2}(i)\right) .
\end{aligned}
$$

Equation (3.29) has a nontrivial solution $\left(c_{0}, c_{1}\right)$ if and only if

$$
\operatorname{Im}(\xi(\rho) \bar{\eta}(\rho))=0 \text {. }
$$

Thus we obtained the following converse to Lemma 3.4.

Lemma 3.5. Let $(p, q, r, \rho)$ be a solution to the system, which consists of integral equations (3.27) and algebraic equation (3.30), such that $\rho>0$ and

$$
A\{p, q, r\} \in L_{2}\left(\mathbb{R}_{+} ; \mathbb{R}^{2}\right) .
$$

Define $\Psi_{0}(z)$ and $\Psi_{1}(z)$ by (3.28), where $c_{0}$ is an arbitrary real constant and $c_{1}$ is determined by (3.29). Let $\Phi_{0}(z)$ and $\Phi_{1}(z)$ be defined by equations 
(3.21) and $f$ be the inverse Laplace transform of $\widehat{f}$, defined in (3.3) with $\lambda=\rho^{2 \alpha}$ and $c_{f}$ as in (3.23). Then the pair $(\lambda, f)$ is a solution to (P).

3.7. Inversion of the Laplace transform. The inversion of Laplace transform, mentioned in Lemma 3.5. can be carried out by integrating over the imaginary axis. In view of (3.3),

$$
f(x)=-\frac{1}{2 \pi i} \int_{-i \infty}^{i \infty}\left(f_{0}(z)+f_{1}(z)\right) d z
$$

where we defined

$$
f_{0}(z):=\frac{\Phi_{0}(z)}{\Lambda(z)} e^{z x} \quad \text { and } \quad f_{1}(z):=\left(\frac{\Phi_{1}(-z)}{\Lambda(z)}+\psi(1)\right) e^{z(x-1)} .
$$

Integrating over suitable contours as in [7, Lemma 5.8] gives

$$
\begin{aligned}
& \frac{1}{2 \pi i} \int_{-i \infty}^{i \infty}\left(f_{0}(z)+f_{1}(z)\right) d z=\operatorname{Res}\left(f_{0}, z_{0}\right)+\operatorname{Res}\left(f_{0},-z_{0}\right) \\
& \quad+\frac{1}{2 \pi i} \int_{0}^{\infty}\left(f_{1}^{+}(t)-f_{1}^{-} 1(t)\right) d t+\frac{1}{2 \pi i} \int_{0}^{\infty}\left(f_{0}^{-}(-t)-f_{0}^{+}(-t)\right) d t .
\end{aligned}
$$

In view of symmetries (3.14),

$$
\begin{gathered}
f_{0}^{-}(-t)-f_{0}^{+}(-t)=-e^{-t x} \Phi_{0}(-t) \frac{2 i \sin (\theta(t)-\alpha \pi)}{\left|\Lambda^{+}(t)\right|}, \\
f_{1}^{+}(t)-f_{1}^{-}(t)=-e^{-t(1-x)} \Phi_{1}(-t) \frac{2 i \sin \theta(t)}{\left|\Lambda^{+}(t)\right|} .
\end{gathered}
$$

The residues are complex conjugates, $\operatorname{Res}\left(f_{0},-z_{0}\right)=\overline{\operatorname{Res}\left(f_{0}, z_{0}\right)}$, and

$$
\operatorname{Res}\left(f_{0}, z_{0}\right)=\frac{\Phi_{0}\left(z_{0}\right)}{\Lambda^{\prime}\left(z_{0}\right)} e^{z_{0} x}=-\frac{1}{2 i} \rho^{1-\alpha} e^{-\frac{\pi}{2} \alpha i} \frac{\sin (\alpha \pi)}{\alpha \pi} \Phi_{0}\left(z_{0}\right) e^{z_{0} x} .
$$

Plugging these expressions into (3.32) we obtain

$$
\begin{aligned}
& f(x)=\operatorname{Re}\left(\frac{1}{i} \rho^{1-\alpha} e^{-\frac{\pi}{2} \alpha i} \frac{\sin (\alpha \pi)}{\alpha \pi} \Phi_{0}\left(z_{0}\right) e^{z_{0} x}\right) \\
& +\frac{1}{\pi} \int_{0}^{\infty} e^{-t(1-x)} \Phi_{1}(-t) \frac{\sin \theta(t)}{\left|\Lambda^{+}(t)\right|} d t+\frac{1}{\pi} \int_{0}^{\infty} e^{-t x} \Phi_{0}(-t) \frac{\sin (\theta(t)-\alpha \pi)}{\left|\Lambda^{+}(t)\right|} d t .
\end{aligned}
$$

3.8. Asymptotic analysis. Similarly to [7, Lemma 5.6], the integral operator in (3.26) can be proved to be a contraction on $L^{2}\left(\mathbb{R}_{+} ; \mathbb{R}^{2}\right)$ for all sufficiently large values of $\rho$ and therefore equations (3.27) have unique solutions with the property (3.31). As in [7, Lemma 5.7], these solutions can 
be seen to satisfy the estimates

$$
\left\|p(z)-\left(\begin{array}{l}
1 \\
0
\end{array}\right)\right\| \vee\left\|q(z)-\left(\begin{array}{l}
0 \\
1
\end{array}\right)\right\| \vee\left\|r(z)-\left(\begin{array}{l}
0 \\
z
\end{array}\right)\right\| \leq C|z|^{-1} \rho^{-1}
$$

with a constant $C$.

3.8.1. Eigenvalues. In view of (3.30) and estimates (3.34),

$$
\xi(\rho) \bar{\eta}(\rho)=(\rho i)^{-1} X_{c 0}(i) \rho^{\alpha}(-i)^{\alpha-1} e^{\rho i} X_{c 0}(i)\left(b_{\alpha}+i\right)(1+R(\rho)),
$$

where $R(\rho)$ can be shown to satisfy $|R(\rho)| \vee\left|R^{\prime}(\rho)\right| \leq C \rho^{-1}$ for some constant $C$. Consequently (3.30) is equivalent to the equations

$$
\begin{aligned}
\rho= & \pi n+\frac{\pi}{2} \alpha-2 \arg \left\{X_{c 0}(i)\right\} \\
& -\arg \left\{b_{\alpha}+i\right\}-\operatorname{atan} \frac{\operatorname{Im}(R(\rho))}{1+\operatorname{Re}(R(\rho))}, \quad n \in \mathbb{Z} .
\end{aligned}
$$

For each sufficiently large $n$ the derivative of the expression in the right hand side with respect to $\rho$ is less than unity. Hence if $n$ is taken large enough, so that, in addition, the operator (3.26) is a contraction, the integro-algebraic system from Lemma 3.5 has the unique solution, obtained by fixed-point iterations. This, in turn, implies that the system (3.24)-(3.25) has the unique solution for all $\rho>0$ large enough, defined by (3.28). Then since $(\mathrm{P})$ has only finitely many eigenvalues on any bounded interval, in view of Lemma 3.4, all but possibly a finite number of eigenvalues correspond to solutions of the system of Lemma 3.5.

In view of (3.35), the algebraic part of these solutions $\rho_{n}$ satisfies

$$
\rho_{n}=\pi n+\frac{\pi}{2} \alpha-2 \arg \left\{X_{c 0}(i)\right\}-\arg \left\{b_{\alpha}+i\right\}+O\left(n^{-1}\right) .
$$

Here the last two quantities have explicit values, see Lemma 3.3 ,

and consequently,

$$
\arg \left(X_{c 0}(i)\right)=-\frac{\pi}{4}(1-\alpha) \quad \text { and } \quad \arg \left(b_{\alpha}+i\right)=\frac{\pi}{2 \alpha},
$$

$$
\rho_{n}=\pi n+\frac{\pi}{2}(1-1 / \alpha)+O\left(n^{-1}\right), \quad n \rightarrow \infty .
$$

Equations (3.35) fix a particular enumeration of solutions to the integroalgebraic system and, therefore, as argued above, of all sufficiently large eigenvalues $\lambda_{n}=\rho_{n}^{2 \alpha}$. Since any bounded interval contains only finitely many eigenvalues, the enumeration that puts all the eigenvalues into the nondecreasing order may differ only by a finite shift. This shift can be identified by comparing (3.36) at $\alpha=1$ to the classical problem (2.3), which shows that, in fact, the two enumerations coincide, that is, (2.1) holds. The technical details of this calibration procedure are the same as in [7, Subsection 5.1.7]. 
3.8.2. Eigenfunctions. When condition (3.30) is satisfied, the equation (3.29) determines the ratio

$$
\begin{aligned}
& c_{1} / c_{0}=-\frac{\operatorname{Re}(\xi \bar{\eta})}{|\eta|^{2}}=-\operatorname{Re}\left(\frac{\xi}{\eta}\right) \\
& =-\frac{\rho_{n}^{-1-\alpha}}{\sqrt{b_{\alpha}^{2}+1}} \cos \left(\rho_{n}-\frac{\pi}{2} \alpha+2 \arg \left\{X_{c 0}(i)\right\}-\arg \left(b_{\alpha}-i\right)\right)\left(1+O\left(\rho_{n}^{-1}\right)\right) \\
& =-\frac{\rho_{n}^{-1-\alpha}}{\sqrt{b_{\alpha}^{2}+1}} \cos \left(\pi n+O\left(n^{-1}\right)\right)\left(1+O\left(n^{-1}\right)\right) \\
& =-\frac{\rho_{n}^{-1-\alpha}}{\sqrt{b_{\alpha}^{2}+1}}(-1)^{n}\left(1+O\left(n^{-1}\right)\right),
\end{aligned}
$$

where we used estimates (3.34). Thus, in view of equation (3.28),

$$
\Psi_{0}\left(i \rho_{n}\right)=c_{0} p_{1}(-i)+c_{1} \rho_{n}^{1+\alpha} b_{\alpha} q_{1}(-i)-c_{1} \rho_{n}^{1+\alpha} r_{1}(-i)=c_{0}\left(1+O\left(n^{-1}\right)\right),
$$

and consequently,

$$
\begin{gathered}
\Phi_{0}\left(z_{0}\right)=\Psi_{0}\left(i \rho_{n}\right) X\left(i \rho_{n}\right)=\Psi_{0}\left(i \rho_{n}\right)\left(i \rho_{n}\right)^{-1} X_{c, 0}(i) \\
=c_{0} \rho_{n}^{-1} e^{-\frac{\pi}{2} i} X_{c 0}(i)\left(1+O\left(n^{-1}\right)\right) .
\end{gathered}
$$

The oscillating term in (3.33) can be now simplified to

$$
\begin{aligned}
& 2 \operatorname{Re}\left(\frac{1}{2 i} \rho^{1-\alpha} e^{-\frac{\pi}{2} \alpha i} \frac{\sin (\alpha \pi)}{\alpha \pi} \Phi_{0}\left(z_{0}\right) e^{z_{0} x}\right) \\
& =\rho_{n}^{1-\alpha} \frac{\sin (\alpha \pi)}{\alpha \pi} \operatorname{Re}\left(e^{i \rho_{n} x-\frac{\pi}{2}(\alpha+1) i} \Phi_{0}\left(i \rho_{n}\right)\right) \\
& =\rho_{n}^{-\alpha} c_{0}\left|X_{c 0}(i)\right| \frac{\sin (\alpha \pi)}{\alpha \pi} \cos \left(\rho_{n} x-\frac{\pi}{2}(\alpha+1)-\frac{\pi}{2}+\arg \left(X_{c 0}(i)\right)\right)\left(1+O\left(n^{-1}\right)\right) \\
& =\rho_{n}^{-\alpha} c_{0}\left|X_{c 0}(i)\right| \frac{\sin (\alpha \pi)}{\alpha \pi} \cos \left(\rho_{n} x-\frac{\pi}{4} \alpha-\frac{5 \pi}{4}\right)\left(1+O\left(n^{-1}\right)\right) \\
& =-\rho_{n}^{-\alpha} c_{0} \sqrt{\alpha} \frac{\sin (\alpha \pi)}{\alpha \pi} \sin \left(\rho_{n} x+\frac{\pi}{4}(1-\alpha)\right)\left(1+O\left(n^{-1}\right)\right),
\end{aligned}
$$

where we used (3.20). The boundary layer terms are treated similarly,

$$
\begin{aligned}
& \frac{1}{\pi} \int_{0}^{\infty} e^{-t(1-x)} \Phi_{1}(-t) \frac{\sin \theta(t)}{\left|\Lambda^{+}(t)\right|} d t \\
& =\rho_{n}^{1-\alpha} \frac{\sin (\alpha \pi)}{\alpha \pi} \frac{\alpha}{\pi} \int_{0}^{\infty} \Phi_{1}\left(-\tau \rho_{n}\right) \frac{\sin \theta_{0}(\tau)}{\gamma_{0}(\tau)} e^{-\rho_{n}(1-x) \tau} d \tau \\
& =(-1)^{n} \frac{c_{0}}{\rho_{n}^{\alpha}} \frac{\sin (\alpha \pi)}{\alpha \pi} \frac{\alpha}{\pi} \int_{0}^{\infty} \frac{\tau^{\alpha}\left(\tau-b_{\alpha}\right)}{\sqrt{b_{\alpha}^{2}+1}} \frac{X_{c 0}(-\tau)}{\tau} \frac{\sin \theta_{0}(\tau)}{\gamma_{0}(\tau)} e^{-\rho_{n}(1-x) \tau} d \tau\left(1+O\left(n^{-1}\right)\right),
\end{aligned}
$$

where $\gamma_{0}(\tau)$ is defined by the equation

$$
\left|\Lambda^{+}\left(\tau \rho_{n}\right)\right|=\rho_{n}^{\alpha} \frac{\pi}{\sin (\alpha \pi)}\left|\tau^{\alpha}-e^{\alpha \pi i} \tau^{-\alpha}\right|=: \rho_{n}^{\alpha} \frac{\pi}{\sin (\alpha \pi)} \gamma_{0}(\tau),
$$


and we used the uniform approximation due to (3.34),

$$
\begin{aligned}
& \Phi_{1}\left(-\tau \rho_{n}\right)=Y\left(-\tau \rho_{n}\right) \Psi_{1}\left(-\tau \rho_{n}\right) \\
& =\left(\tau \rho_{n}\right)^{\alpha-1} X_{c}\left(-\tau \rho_{n}\right)\left(\rho_{n}^{-\alpha} c_{0} p_{2}(\tau)+c_{1} \rho_{n} b_{\alpha} q_{2}(\tau)-c_{1} \rho_{n} r_{2}(\tau)\right) \\
& =-c_{0} \rho_{n}^{-1}(-1)^{n} \frac{1}{\sqrt{b_{\alpha}^{2}+1}} \tau^{\alpha-1} X_{c 0}(-\tau)\left(b_{\alpha}-\tau\right)\left(1+O\left(n^{-1}\right)\right) .
\end{aligned}
$$

Similarly,

$$
\begin{aligned}
& \frac{1}{\pi} \int_{0}^{\infty} e^{-t x} \Phi_{0}(-t) \frac{\sin (\theta(t)-\alpha \pi)}{\left|\Lambda^{+}(t)\right|} d t \\
& =\rho_{n} \frac{1}{\pi} \int_{0}^{\infty} e^{-\rho_{n} x \tau} \Phi_{0}\left(-\rho_{n} \tau\right) \frac{\sin \left(\theta\left(\rho_{n} \tau\right)-\alpha \pi\right)}{\left|\Lambda^{+}\left(\rho_{n} \tau\right)\right|} d \tau \\
& =\rho_{n}^{1-\alpha} \frac{\sin (\alpha \pi)}{\pi \alpha} \frac{\alpha}{\pi} \int_{0}^{\infty} e^{-\rho_{n} x \tau} \Phi_{0}\left(-\rho_{n} \tau\right) \frac{\sin \left(\theta_{0}(\tau)-\alpha \pi\right)}{\gamma_{0}(\tau)} d \tau \\
& =-c_{0} \rho_{n}^{-\alpha} \frac{\sin (\alpha \pi)}{\pi \alpha} \frac{\alpha}{\pi} \int_{0}^{\infty} \frac{X_{c 0}(-\tau)}{\tau} \frac{\sin \left(\theta_{0}(\tau)-\alpha \pi\right)}{\gamma_{0}(\tau)} e^{-\rho_{n} x \tau} d \tau\left(1+O\left(n^{-1}\right)\right),
\end{aligned}
$$

where we used the approximation

$$
\begin{aligned}
\Phi_{0}\left(-\rho_{n} \tau\right)= & X\left(-\rho_{n} \tau\right) \Psi_{0}\left(-\rho_{n} \tau\right) \\
& =X\left(-\rho_{n} \tau\right)\left(c_{0} p_{1}(\tau)+c_{1} \rho_{n}^{1+\alpha} b_{\alpha} q_{1}(\tau)-c_{1} \rho_{n}^{1+\alpha} r_{1}(\tau)\right) \\
& =-c_{0} \rho_{n}^{-1} \tau^{-1} X_{c 0}(-\tau)\left(1+O\left(n^{-1}\right)\right) .
\end{aligned}
$$

Assembling all parts in (3.33) together and normalising up to the unit norm, we arrive at (2.2) with

$$
\begin{aligned}
& \Upsilon_{0}(\tau)=\frac{\sqrt{2 \alpha}}{\pi} \frac{X_{c 0}(-\tau)}{\tau} \frac{\sin \left(\theta_{0}(\tau)-\alpha \pi\right)}{\gamma_{0}(\tau)} \\
& \Upsilon_{1}(\tau)=\frac{\sqrt{2 \alpha}}{\pi} \frac{\tau^{\alpha}\left(b_{\alpha}-\tau\right)}{\sqrt{b_{\alpha}^{2}+1}} \frac{X_{c 0}(-\tau)}{\tau} \frac{\sin \theta_{0}(\tau)}{\gamma_{0}(\tau)},
\end{aligned}
$$

where, cf. (3.37) and Lemma 3.2 (c),

$\gamma_{0}(\tau)=\left(\tau^{2 \alpha}-2 \cos (\alpha \pi)+\tau^{-2 \alpha}\right)^{1 / 2}$ and $\quad \theta_{0}(\tau)=-\operatorname{atan} \frac{\sin (\alpha \pi)}{\tau^{2 \alpha}-\cos (\alpha \pi)}$

and, cf. Lemma $3.3, b_{\alpha}=\cot \left(\frac{\pi}{2 \alpha}\right)$ and

$$
X_{c 0}(-\tau)=\exp \left(\frac{1}{\pi} \int_{0}^{\infty} \frac{\theta_{0}(t)}{t+\tau} d t\right) .
$$




\section{Conclusions}

This paper constructs an asymptotic approximation of solutions to a fractional Sturm-Liouville problem of a particular type. The obtained eigenvalues asymptotics (2.1) is exact up to the second order, with an estimate for the residual. This precision turns out to be sufficient for uniform approximation (2.2) of the eigenfunctions, which sheds some light on their general structure. The suggested method is based on reduction to a Hilbert boundary value problem. While the details are worked out in a specific problem, the technique is expected to be applicable to other fractional eigenproblems of interest.

\section{Acknowledgements}

P. Chigansky's research was funded by ISF 1383/18 grant.

\section{References}

[1] M.S. Birman, M.Z. Solomyak, Spectral asymptotics of weakly polar integral operators. Izv. AN SSSR 34 (1970), 1143-1158; DOI: 10.1070/im1970v004n05abeh000948.

[2] T. Blaszczyk, M. Ciesielski, M. Klimek, J. Leszczynski, Numerical solution of fractional oscillator equation. Appl. Math. Comput. 218, No 6 (2011), 2480-2488; DOI: 10.1016/j.amc.2011.07.062.

[3] T. Blaszczyk, M. Ciesielski, Numerical solution of fractional SturmLiouville equation in integral form. Fract. Calc. Appl. Anal. 17, No 2 (2014), 307-320; DOI: 10.2478/s13540-014-0170-8.

[4] T. Blaszczyk, M. Ciesielski, Fractional oscillator equation: analytical solution and algorithm for its approximate computation. J. Vib. Control 22, No 8 (2016), 2045-2052; DOI: 10.1177/1077546314566836.

[5] J.C. Bronski, Asymptotics of Karhunen-Loeve eigenvalues and tight constants for probability distributions of passive scalar transport. Comm. Math. Phys. 238, No 3 (2003), 563-582; DOI: 10.1007/s00220003-0835-3.

[6] P. Chigansky, M. Kleptsyna, D. Marushkevych, Mixed fractional Brownian motion: a spectral take. J. Math. Anal. Appl. 482, No 2 (2020), 123558; DOI: 10.1016/j.jmaa.2019.123558.

[7] P. Chigansky, Marina Kleptsyna, Exact asymptotics in eigenproblems for fractional Brownian covariance operators. Stochastic Process. Appl. 128, No 6 (2018), 2007-2059; DOI: 10.1016/j.spa.2017.08.019.

[8] M. Dehghan, A. B. Mingarelli, Fractional Sturm-Liouville eigenvalue problems, I. Rev. R. Acad. Cienc. Exactas Fis. Nat. Ser. A Mat. RACSAM 114, No 2 (2020), Paper No. 46, 15; DOI: 10.1007/s13398-01900756-8. 
[9] M-H. Derakhshan, A. Ansari, Fractional Sturm-Liouville problems for Weber fractional derivatives. Int. J. Comput. Math. 96, No 2 (2019), 217-237; DOI: 10.1080/00207160.2018.1425797.

[10] F.D. Gakhov, Boundary Value Problems. Dover Publications, Inc., New York (1990).

[11] A.G. Gibbs, Analytical solutions of the neutron transport equation in arbitrary convex geometry. J. Mathematical Phys. 10 (1969), 875-890; DOI: $10.1063 / 1.1664917$.

[12] H. Jin, W. Liu, Eigenvalue problem for fractional differential operator containing left and right fractional derivatives. Adv. Difference Equ. (2016), Paper No. 246, 12; DOI: 10.1186/s13662-016-0950-z.

[13] A.A. Kilbas, H M. Srivastava, J J. Trujillo, Theory and Applications of Fractional Differential Equations. Elsevier Sci. B.V., Amsterdam, (2006).

[14] M.L. Kleptsyna, D.A. Marushkevych, P.Yu. Chigansky, Asymptotic accuracy in estimation of a fractional signal in a small white noise. Automation and Remote Control 81, No 3 (2020), 411-429; DOI: 10.1134/S0005117920030030.

[15] M. Klimek, O.P. Agrawal, Fractional Sturm-Liouville problem. Comput. Math. Appl. 66, No 5 (2013), 795-812; DOI: 10.1016/j.camwa.2012.12.011.

[16] M. Klimek, M. Blasik, Regular fractional Sturm-Liouville problem with discrete spectrum: Solutions and applications. In: ICFDA'14 International Conference on Fractional Differentiation and Its Applications (2014), 1-6; DOI: doi: 10.1109/ICFDA.2014.6967383.

[17] M. Klimek, T. Odzijewicz, A.B. Malinowska, Variational methods for the fractional Sturm-Liouville problem. J. Math. Anal. Appl. 416, No 1 (2014), 402-426; DOI: 10.1016/j.jmaa.2014.02.009.

[18] M. Klimek, Homogeneous Robin boundary conditions and discrete spectrum of fractional eigenvalue problem. Fract. Calc. Appl. Anal., 22, No 1 (2019), 78-94; DOI: 10.1515/fca-2019-0005;

[19] M. Klimek, M. Blasik. Regular Sturm-Liouville problem with Riemann-Liouville derivatives of order in $(1,2)$ : discrete spectrum, solutions and applications. In: Advances in Modelling and Control of Non-integer Order Systems, Vol. 320 of Lect. Notes Electr. Eng., Springer, Cham (2015), 25-36; DOI: 10.1007/978-3-319-09900-2.

[20] M. Klimek, M. Ciesielski, T. Blaszczyk, Exact and numerical solutions of the fractional Sturm-Liouville problem. Fract. Calc. Appl. Anal. 21, No 1 (2018), 45-71; DOI: 10.1515/fca-2018-0004; 
[21] J. Li, J. Qi, Eigenvalue problems for fractional differential equations with right and left fractional derivatives. Appl. Math. Comput. 256 (2015), 1-10; DOI: 10.1016/j.amc.2014.12.146.

[22] J. Li, J. Qi, Note on a nonlocal Sturm-Liouville problem with both right and left fractional derivatives. Appl. Math. Lett. 97 (2019), 1419; DOI: 10.1016/j.aml.2019.05.011.

[23] W.V. Li, Q.M. Shao, Gaussian processes: inequalities, small ball probabilities and applications. In: Stochastic Processes: Theory and Methods, Vol. 19 of Handbook of Statist., North-Holland, Amsterdam (2001), 533-597; DOI: 10.1016/S0169-7161(01)19019-X.

[24] A.I. Nazarov, Spectral asymptotics for a class of integro-differential equations arising in the theory of fractional Gaussian processes. To appear in: Commun. Contemp. Math., arXiv:1908.10299 (2019) Preprint.

[25] R. Ozarslan, E. Bas, D. Baleanu, Representation of solutions for Sturm-Liouville eigenvalue problems with generalized fractional derivative. Chaos 30, No 3 (2020), 033137, 11 pp.; DOI: 10.1063/1.5131167.

[26] B. V. Pal'cev, Asymptotic behavior of the spectrum and eigenfunctions of convolution operators on a finite interval with the kernel having a homogeneous Fourier transform. Dokl. Akad. Nauk SSSR, 218 (1974), $28-31$.

[27] B. V. Pal'tsev, Asymptotics of the spectrum of integral convolution operators on a finite interval with homogeneous polar kernels. Izv. Ross. Akad. Nauk Ser. Mat. 67, No 4 (2003), 67-154; DOI: 10.1070/IM2003v067n04ABEH000443.

[28] J. Qi, S. Chen, Eigenvalue problems of the model from nonlocal continuum mechanics. J. Math. Phys. 52, No 7 (2011), 073516, 14 pp.; DOI: $10.1063 / 1.3610673$.

[29] S. Ukai, Asymptotic distribution of eigenvalues of the kernel in the Kirkwood-Riseman integral equation. J. Mathematical Phys. 12 (1971), 83-92; DOI: 10.1063/1.1665491.

[30] M. Zayernouri, G E. Karniadakis, Fractional Sturm-Liouville eigenproblems: theory and numerical approximation. J. Comput. Phys. 252 (2013), 495-517; DOI: 10.1016/j.jcp.2013.06.031.

${ }^{1}$ Department of Statistics, The Hebrew University of Jerusalem Mount Scopus, Jerusalem 91905, ISRAEL

e-mail: pchiga@mscc.huji.ac.il (Corresponding author)

Received: June 12, 2020, Revised: 2021

2 Laboratoire de Statistique et Processus

Le Mans Université, FRANCE

e-mail: marina.kleptsyna@univ-lemans.fr 